\title{
Perfil de segurança de medicamentos antidiabéticos para uso em pacientes idosos com doença renal crônica
}

\author{
Ana Cláudia Oliveira Costa*, Alba Otoni ${ }^{\star *}$, Aisha Aguiar Morais ${ }^{\star * *}$, André Oliveira Baldoni ${ }^{\star \star * *}$
}

\section{Resumo}

A prevalência de diabetes mellitus (DM) aumenta com o passar da idade, de modo que $21,6 \%$ dos brasileiros com 65 anos ou mais apresentam a doença. DM é uma das causas mais frequentes de doença renal crônica (DRC), sendo que o quadro clínico pode ser agravado com o aumento da idade do paciente. Existem diversas classes de medicamentos antidiabéticos disponíveis, no entanto, a escolha do tratamento para pacientes com DRC deve ser criteriosa, especialmente em idosos. Os objetivos do presente estudo foram analisar o perfil de segurança dos antidiabéticos para uso em pacientes idosos com DRC e categorizá- los quanto ao cuidado na escolha. Para busca das informações, utilizaram-se o Micromedex $^{\circledR}$ e artigos publicados nas bases de dados PubMed e SciELO até novembro de 2015. Realizou-se a análise individual do perfil farmacocinético dos antidiabéticos, as possíveis contraindicações e restri- ções de uso em caso de DRC. Observou-se que a clorpropramida deve ser evitada por idosos com DRC. Outros devem ser utilizados com cautela, com base na taxa de filtração glomerular (TFG) e clearance de creatinina: nateglinida, liraglutida, exenatida, sitagliptina, vildagliptina, saxagliptina, metformina, pioglitazona, acarbose, insulina e a classe dos inibidores do cotransportador de sódio e glicose 2 (iSGLT2). Opções consideradas seguras são gliclazida, glipizida, repaglinida e linagliptina. Diante do exposto, entende-se que a escolha do antidiabético para idosos com DRC deve ser baseada no perfil farmacocinético dos medicamentos e em parâmetros clínicos e laboratoriais do paciente.

Palavras-chave: Diabetes mellitus. Falência renal crônica. Hipoglicemiantes. Nefropatias diabéticas.

* Farmacêutica pela Universidade Federal de São João Del-Rei, Campus Centro-Oeste Dona Lindu. Endereço para correspondência: Rua Sebastião Gonçalves Coelho 400, sala 308.3D, Bairro Chanadour, CEP 35.501296, Divinópolis, MG, Brasil. E-mail: anaclaudiacosta04@gmail.com

** Doutora em Ciências da Saúde pela Faculdade de Medicina da Universidade Federal de Minas Gerais. Professora Adjunta na Universidade Federal de São João Del-Rei, Campus Centro-Oeste Dona Lindu, Curso de Enfermagem - Doença Renal Crônica. E-mail: albaotoni@ufsj.edu.br

*** Médica endocrinologista, especialista em nutrição clínica pelo Ganep Nutrição Humana, mestre em Ciências da Saúde e professora de Clínica Médica na Universidade Federal de São João Del Rei, Campus Centro-Oeste. E-mail: draaishacorpoealma@gmail.com

***** Doutor em Ciências Farmacêuticas pela Faculdade de Ciências Farmacêuticas da Universidade de São Paulo, Campus de Ribeirão Preto. Orientador no Programa de Pós-Graduação em Ciências da Saúde e do Programa de Pós-Graduação em Ciências Farmacêuticas da Universidade Federal de São João Del-Rei, Campus Centro-Oeste Dona Lindu, Curso de Farmácia. E-mail: andrebaldoni@ufsj.edu.br

$\rightarrow$ http://dx.doi.org/10.5335/rbceh.v13i2.5754

Recebido em: 20/02/2016. Aceito em: 25/07/2016. 


\section{Introdução}

Atualmente, o Brasil passa por um processo de transição demográfica, em que se observa a diminuição na taxa de natalidade e o aumento do número de idosos. Além disso, ocorre a transição epidemiológica, caracterizada por uma alteração no perfil de morbimortalidade, na qual há substituição das doenças infectocontagiosas por doenças crônicas não transmissíveis, como hipertensão arterial sistêmica e diabetes mellitus, que, por sua vez, podem acarretar complicações clínicas que prejudicam a qualidade de vida dos pacientes (BORGES; CAMPOS; SILVA, 2015).

O DM configura-se como epidemia mundial e sua prevalência no Brasil aumenta. Um estudo realizado em 26 capitais e no Distrito Federal revelou que $21,6 \%$ dos pacientes com 65 anos ou mais de idade apresentam DM (SOCIEDADE BRASILEIRA DE ENDOCRINOLOGIA E METABOLOGIA, 2011). Durante a evolução da doença, ocorrem alterações patológicas crônicas que podem acometer o sistema vascular, os nervos, a pele e o cristalino (GREENSPAN; STREWLER, 2006). As lesões microvasculares levam a um aumento na excreção urinária de proteína e, mais tardiamente, ao aumento da ureia e creatinina no sangue. A perda da função renal é lenta e progressiva e pode ser detectada por meio de exames laboratoriais (estimativa de TFG, albuminúria e proteinúria) e de imagem (tamanho, forma, ecogenicidade e simetria dos rins, além de obstrução/ estenose vascular e uropatia obstrutiva)
(SOCIEDADE BRASILEIRA DE NEFROLOGIA, 2007).

É importante ressaltar que o próprio processo de envelhecimento gera alterações anatômicas, como a diminuição do peso e tamanho dos rins, e alterações fisiológicas, ocasionando diminuição do fluxo plasmático renal, que juntamente com os quadros de descompensação diabética podem favorecer o desenvolvimento de doença renal crônica (DRC) (LEMOS et al., 2008; TONELLI; RIELLA, 2014).

Segundo as Diretrizes para avaliação e manuseio da doença renal na prática clínica (KIDNEY DISEASE IMPROVEMENT GLOBAL OUTCOMES, 2012), a DRC pode ser classificada em categorias, conforme a taxa de filtração estimada: Grupo $1 \mathrm{TFG}>89 \mathrm{~mL} \backslash \min \backslash 1.73 \mathrm{~m}^{2}$, Grupo 2 60-89 $\mathrm{mL} \backslash \min \backslash 1.73 \mathrm{~m}^{2}$, Grupo 3a $45-60 \mathrm{~mL} \backslash \min \backslash 1.73 \mathrm{~m}^{2}$, Grupo $3 \mathrm{~b}$ 30-44 $\mathrm{mL} \backslash \min \backslash 1.73 \mathrm{~m}^{2}$, Grupo 4 15-29 $\mathrm{mL} \backslash \min \backslash 1.73 \mathrm{~m}^{2}$ e Grupo $5<15 \mathrm{~mL} \backslash$ $\min \backslash 1.73 \mathrm{~m}^{2}$. O diagnóstico de DRC é confirmado nas categorias a partir do Grupo 3a e a gravidade do comprometimento renal é considerada de forma crescente a partir da categoria Grupo 1.

Para tratamento do DM, além da educação em saúde e da mudança no estilo de vida do paciente, existem disponíveis no Brasil várias classes de medicamentos, como: secretagogos de insulina (sulfonilureias e glinidas), sensibilizadores da ação da insulina (tiazolidinodionas), redutores da gliconeogênese (biguanidas), redutores da velocidade de absorção de glicídios (inibidores da a-glicosidase), incretinomiméticos (inibidores da dipeptidil peptidase 
4 - iDPP4) e análogos de GLP-1 (glucagon-likepeptide 1), inibidores da reabsorção de glicose (inibidores do cotransportador de sódio e glicose 2 - iSGLT2) e insulinas (SOCIEDADE BRASILEIRA DE DIABETES, 2015; OBRELI NETO; BALDONI; GUIDONI, 2013).

Diante do exposto, o controle da glicemia, particularmente em pacientes em estágio avançado de DRC (grupos 3 a 5), torna-se um desafio, visto que a condição impõe limitação ao uso de alguns antidiabéticos. A redução da função renal pode levar ao acúmulo do medicamento e/ou de metabólitos ativos no organismo, causando quadros graves de hipoglicemia, alterações metabólicas, arritmia cardíaca, hipoperfusão vascular e dano cerebral irreversível (BASTOS; BREGMAN; KIRSZTAJN, 2010; SHIH et al., 2015). Torna-se essencial investigar formas de racionalizar o uso dos medicamentos que consigam controlar a glicemia e, com isso, retardar a progressão da DRC, mas com o mínimo de efeitos adversos (TONELLI; RIELLA, 2014).

O objetivo do presente estudo é analisar o perfil de segurança dos antidiabéticos para uso em pacientes idosos com DRC bem como categorizá-los em relação aos cuidados necessários para escolha desses medicamentos.

\section{Metodologia}

Este é um estudo de revisão narrativa da literatura, que incluiu artigos publicados até novembro de 2015. Para esta pesquisa, foram utilizados artigos sobre antidiabéticos em pacientes com DRC publicados nas bases de dados PubMed, SciELO e Micromedex ${ }^{\circledR}$ (MICROME$\left.\mathrm{DEX}^{\circledR}, 2015\right)$. Em todas essas fontes de informações, buscaram-se artigos de todas as classes de antidiabéticos disponíveis no Brasil até novembro de 2015: sulfonilureias, glinidas, biguanidas, tiazolidinodionas, inibidores da a-glicosidase, análogos de GLP-1, iDPP-4, iSGLT2 e insulina (SOCIEDADE BRASILEIRA DE DIABETES, 2015; OBRELI NETO; BALDONI; GUIDONI, 2013).

Após a análise dos parâmetros farmacocinéticos, de riscos de eventos adversos, contraindicações e restrições de uso de cada medicamento em idosos com DRC, os antidiabéticos foram classificados em três categorias:

a) uso não recomendado;

b) uso restrito, a depender dos valores do clearance e/ou a TFG do paciente;

c) medicamentos que podem ser utilizados em idosos com DRC (sem necessidade de ajuste de dose).

Para essa classificação, levou-se em consideração a necessidade de um bom funcionamento renal (TFG, clearance de creatinina e creatinina sérica) para efetividade e/ou segurança do medicamento e a possibilidade de acúmulo de metabólitos ativos e/ou do medicamento na forma inalterada que podem aumentar o risco de hipoglicemia. Após essa categorização, os resultados, com as respectivas justificativas, foram analisados por três juízes/expertises no assunto, professores/ pesquisadores que atuam na prática clínica com pacientes que apresentam DM e/ou DRC. A última fase foi a discussão 
para obtenção de consenso entre os juízes avaliadores.

\section{Resultados}

$\mathrm{Na}$ Tabela 1, apresentam-se as classes dos antidiabéticos, com os parâmetros farmacocinéticos úteis para $o$ manejo clínico do paciente com DRC e a conduta sugerida em caso de paciente nessa condição. Em relação aos achados, observou-se que a clorpropramida deve ser evitada por idosos com DRC. Outros devem ser utilizados com cautela, de acordo com o clearance e a taxa de TFG, como nateglinida, liraglutida, exenatida, sitagliptina, vildagliptina, saxagliptina, metformina, pioglitazona, acarbose, insulina e os iSGLT2. Opções consideradas seguras são: gliclazida, glipizida, repaglinida e linagliptina.

Tabela 1 - Classes, principais fármacos antidiabéticos, farmacocinética e conduta frente ao paciente com doença renal crônica (DRC)

\begin{tabular}{|c|c|c|c|}
\hline $\begin{array}{c}\text { Classe, fármaco } \\
\text { e } t_{1 / 2}\end{array}$ & $\begin{array}{l}\text { Via de } \\
\text { excreção }\end{array}$ & Conduta em caso de DRC & $\begin{array}{c}\text { Doses usuais } \\
\text { (em pacientes sem DRC) }\end{array}$ \\
\hline \multicolumn{4}{|l|}{ Sulfonilureias } \\
\hline $\begin{array}{l}\text { Clorpropamida } \\
25 \text { a } 48 \text { horas }\end{array}$ & $\begin{array}{l}\text { Renal: } 80 \%-90 \% \\
(10 \%-30 \% \text { na forma inalterada) }\end{array}$ & $\begin{array}{l}\text { Clearance <50 mL/min "Não } \\
\text { usar" } \\
\text { Clearance entre } 50 \mathrm{~mL} / \mathrm{min}-70 \\
\mathrm{~mL} / \mathrm{min} \text { "Reduzir a dose em } 50 \% \text { " } \\
\text { Hemodiálise, diálise peritoneal } \\
\text { e terapia de substituição renal } \\
\text { "Não usar" }\end{array}$ & $\begin{array}{l}\text { Oral, } 100 \mathrm{mg} \text { a } 125 \mathrm{mg} \\
\text { uma vez ao dia }\end{array}$ \\
\hline $\begin{array}{l}\text { Glibenclamida } \\
5 \text { a } 10 \text { horas }\end{array}$ & $\begin{array}{l}\text { Renal: } 50 \% \\
\text { Biliar: } 50 \%\end{array}$ & $\begin{array}{l}\text { Clearance }<50 \mathrm{~mL} / \mathrm{min} \text { "Não } \\
\text { usar" } \\
\text { Creatinina }>2 \mathrm{mg} / \mathrm{dL} \text { "Necessário } \\
\text { ajuste de dose" }\end{array}$ & $\begin{array}{l}\text { Oral, } 1,25 \mathrm{mg} \text { uma vez } \\
\text { ao dia }\end{array}$ \\
\hline $\begin{array}{l}\text { Glimepirida } \\
5 \text { a } 9,2 \text { horas }\end{array}$ & $\begin{array}{l}\text { Renal: } 60 \% \\
\text { (80-90\% são metabólitos) } \\
\text { Fecal: } 40 \% \\
\text { (70\% são metabólitos) }\end{array}$ & Dose inicial de $4 \mathrm{mg} / \mathrm{dia}$ & $\begin{array}{l}\text { Oral, } 4 \mathrm{mg} / \mathrm{dia} \text { uma vez } \\
\text { ao dia }\end{array}$ \\
\hline $\begin{array}{l}\text { Glipizida } \\
2 \text { a } 5 \text { horas }\end{array}$ & $\begin{array}{l}\text { Renal: } 90 \% \\
\text { (3\%-9\% na forma inalterada e } \\
80 \% \text { são metabólitos) } \\
\text { Fecal: } 10 \% \text { (menos de } 10 \% \\
\text { inalterada) }\end{array}$ & $\begin{array}{l}\text { Aconselha-se a dose de } 2,5 \mathrm{mg} \\
\text { para evitar hipoglicemia }\end{array}$ & $\begin{array}{l}\text { Oral, 2,5 mg uma vez ao } \\
\text { dia }\end{array}$ \\
\hline $\begin{array}{l}\text { Gliclazida } \\
8 \text { a } 12 \text { horas }\end{array}$ & $\begin{array}{l}\text { Renal } 60 \%-80 \% \\
(1 \%-20 \% \text { na forma inalterada o }\end{array}$ & $\begin{array}{l}\text { Não é necessário ajuste de dose } \\
\text { na doença renal, no entanto, de- }\end{array}$ & $\begin{array}{l}\text { Liberação imediata Oral, } \\
80 \text { mg duas vezes ao dia }\end{array}$ \\
\hline & $\begin{array}{l}\text { restante como metabólitos) } \\
\text { Fecal: } 20 \%\end{array}$ & ve-se evitar nas categorias 4 e 5 . & $\begin{array}{l}\text { Liberação modificada } \\
\text { Oral, } 30 \mathrm{mg} \text { uma vez ao } \\
\text { dia }\end{array}$ \\
\hline
\end{tabular}


Perfil de segurança de medicamentos antidiabéticos para uso em pacientes idosos com doença renal crônica

cont...

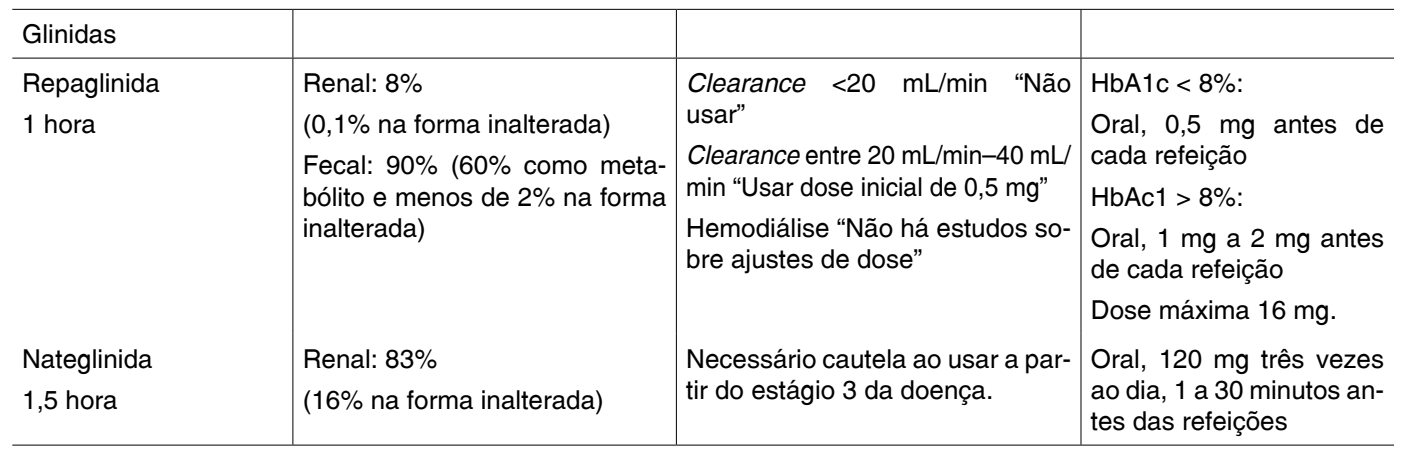

Inibidor da a-glicosidase

\begin{tabular}{l|l|l|l}
\hline $\begin{array}{l}\text { Acarbose } \\
2 \text { horas }\end{array}$ & $\begin{array}{l}\text { Renal: } 34 \% \\
\text { Fecal: } 51 \%\end{array}$ & $\begin{array}{l}\text { Clearance }<29 \mathrm{~mL} / \mathrm{min} \text { "Não } \\
\text { usar" } \\
\text { Creatinina }>2 \mathrm{mg} / \mathrm{dL} \text { "Não usar" } 25 \mathrm{mg} \text { a } 50 \mathrm{mg} \text { junto } \\
\text { com cada refeição principal } \\
\text { Dose máxima em pacien- } \\
\text { tes de } \leq 60 \mathrm{~kg} 50 \mathrm{mg} \text { três } \\
\text { vezes ao dia } \\
\geq 60 \mathrm{~kg} 100 \mathrm{mg}, \mathrm{três} \mathrm{ve-} \\
\text { zes ao dia }\end{array}$ \\
\hline
\end{tabular}

Biguanida

\begin{tabular}{|c|c|c|c|}
\hline $\begin{array}{l}\text { Metformina } \\
1,5 \text { a } 6,2 \text { horas }\end{array}$ & Renal: 90\% & $\begin{array}{l}\text { TFG entre } 30 \mathrm{~mL} / \mathrm{min} / 1,73 \mathrm{~m}^{2}-60 \\
\text { "Reduzir a dose pela metade" } \\
\mathrm{TFG}<30 \mathrm{~mL} / \mathrm{min} / 1,73 \mathrm{~m}^{2} \text { "Não usar" } \\
\text { Creatinina }>1,5 \mathrm{mg} / \mathrm{dL} \text { em ho- } \\
\text { mens e }>1,4 \mathrm{mg} / \mathrm{dL} \text { em mulheres } \\
\text { "Não usar" } \\
\text { Maiores de } 80 \text { anos "Não usar a } \\
\text { menos que seja descartada a dis- } \\
\text { função renal" }\end{array}$ & $\begin{array}{l}\text { Inicial: oral, } 500 \mathrm{mg} \text { duas } \\
\text { vezes ao dia ou } 850 \mathrm{mg} \\
\text { uma vez ao dia } \\
\text { Dose máxima } 2.550 \mathrm{mg} \\
\text { ao dia }\end{array}$ \\
\hline
\end{tabular}

Glitazona

\begin{tabular}{|c|c|c|c|}
\hline $\begin{array}{l}\text { Pioglitazona } \\
16 \text { a } 24 \text { horas }\end{array}$ & $\begin{array}{l}\text { Renal: } 15 \%-30 \% \text { (como meta- } \\
\text { bólitos) } \\
\text { Fecal: (como metabólitos ou } \\
\text { forma inalterada) }\end{array}$ & $\begin{array}{l}\text { Não é necessário ajuste de dose } \\
\text { por não induzir a hipoglicemia, no } \\
\text { entanto, deve ser utilizada com } \\
\text { cautela, devido a suas reações } \\
\text { adversas. }\end{array}$ & $\begin{array}{l}\text { Oral, } 15 \mathrm{mg} \text { a } 30 \mathrm{mg} \text { uma } \\
\text { vez ao dia }\end{array}$ \\
\hline
\end{tabular}

\section{Análogos de GLP-1 (glucagon-likepeptide 1)}

\begin{tabular}{|c|c|c|c|}
\hline $\begin{array}{l}\text { Exenatida } \\
2,4 \text { horas }\end{array}$ & Renal & $\begin{array}{l}\text { Clearance }<30 \mathrm{~mL} / \mathrm{min} \text { "Não usar" } \\
\text { Clearance } 30 \mathrm{~mL} / \mathrm{min}-50 \mathrm{~mL} / \mathrm{min} \\
\text { "Usar com cautela" } \\
\text { Clearance }>50 \mathrm{~mL} / \mathrm{min} \text { "Não é } \\
\text { necessário ajuste de dose" }\end{array}$ & $\begin{array}{l}\text { Liberação imediata: Sub- } \\
\text { cutânea, } 5 \text { mcg duas } \\
\text { vezes ao dia, } 60 \text { minu- } \\
\text { tos antes das refeições, } \\
\text { com cerca de } 6 \text { horas de } \\
\text { intervalo. Com base na } \\
\text { resposta a dose pode ser } \\
\text { aumentada para } 10 \text { mcg } \\
\text { duas vezes ao dia, após } \\
\text { um mês. } \\
\text { Liberação prolongada: } \\
\text { Subcutânea, } 2 \text { mg uma } \\
\text { vez por semana. }\end{array}$ \\
\hline
\end{tabular}


Ana Cláudia Oliveira Costa et al.

cont...

\begin{tabular}{|c|c|c|c|}
\hline $\begin{array}{l}\text { Liraglutida } \\
13 \text { horas }\end{array}$ & $\begin{array}{l}\text { Nenhum órgão específico é } \\
\text { responsável pela excreção. } \\
\text { Renal: } 6 \% \text { (como metabólito) } \\
\text { Fecal: } 5 \% \text { (como metabólito) }\end{array}$ & $\begin{array}{l}\text { Pode ser usado em pacientes } \\
\text { com doença renal nos estágios } \\
1 \text { e } 2 \text { da doença sem ajuste de } \\
\text { dose. } \\
\text { Não há informações naqueles } \\
\text { em estágio } 3 \text { a } 5 \text { da doença. Cle- } \\
\text { arance }<30 \mathrm{~mL} / \mathrm{min} \text { "Utilizar com } \\
\text { cautela" }\end{array}$ & $\begin{array}{l}\text { Subcutânea, } 0,6 \text { mg uma } \\
\text { vez ao dia por uma se- } \\
\text { mana; aumentar para } 1,2 \\
\text { mg uma vez ao dia; po- } \\
\text { dendo aumentar para } 1,8 \\
\text { mg ao dia, dependendo } \\
\text { da resposta glicêmica. }\end{array}$ \\
\hline $\begin{array}{l}\text { Lixisenatide } \\
2,7 \text { a } 4,3 \text { horas }\end{array}$ & Renal & $\begin{array}{l}\text { Clearance }<30 \mathrm{~mL} / \mathrm{min} \text { "Não usar" } \\
\text { Clearance entre } 30 \text { e } 50 \mathrm{~mL} / \mathrm{min}- \\
50 \mathrm{~mL} / \mathrm{min} \text { "Utilizar com cautela" } \\
\text { Clearance }>50 \mathrm{~mL} / \mathrm{min} \text { "Não é ne- } \\
\text { cessário ajuste de dose" }\end{array}$ & $\begin{array}{l}\text { Inicial: subcutânea, } 10 \\
\text { mcg uma vez ao dia por } \\
14 \text { dias. } \\
\text { Manutenção: } 20 \text { mcg } \\
\text { uma vez ao dia, come- } \\
\text { çando no } 15^{\circ} \text { dia. } \\
\text { "Administrar até } 60 \text { minu- } \\
\text { tos antes da refeição". }\end{array}$ \\
\hline \multicolumn{4}{|l|}{ Inibidores de DPP-4 } \\
\hline $\begin{array}{l}\text { Sitagliptina } \\
12,4 \text { horas }\end{array}$ & $\begin{array}{l}\text { Renal: } 87 \% \\
\text { (79\% na forma inalterada) } \\
\text { Fecal: } 13 \%\end{array}$ & $\begin{array}{l}\text { Clearance }<30 \mathrm{~mL} / \mathrm{min} \text {, hemodiá- } \\
\text { lise e diálise peritoneal "Usar } 25 \\
\mathrm{mg} / \text { dia" } \\
\text { Clearance entre } 30 \mathrm{~mL} / \mathrm{min} \text { - } \\
-50 \mathrm{~mL} / \mathrm{min} \text { "Usar } 50 \mathrm{mg} / \text { dia" } \\
\text { Clearance }>50 \mathrm{~mL} / \mathrm{min} \text { "Não é } \\
\text { necessário ajuste de dose" } \\
\text { Em idosos sem doença renal } \\
\text { "Não é necessário ajuste de dose" }\end{array}$ & $\begin{array}{l}\text { Oral, } 100 \mathrm{mg} \text { uma vez } \\
\text { ao dia }\end{array}$ \\
\hline $\begin{array}{l}\text { Vildagliptina } \\
2 \text { a } 3 \text { horas }\end{array}$ & $\begin{array}{l}\text { Renal: } 85 \% \\
\text { Fecal: } 15 \%\end{array}$ & $\begin{array}{l}\text { Clearance }<50 \mathrm{~mL} / \mathrm{min} \text { "Neces- } \\
\text { sário ajuste de dose" } \\
\text { Clearance }>50 \mathrm{~mL} / \mathrm{min} \text { "Não é } \\
\text { necessário ajuste de dose" }\end{array}$ & $\begin{array}{l}\text { Oral, } 50 \mathrm{mg} \text { uma vez ao } \\
\text { dia }\end{array}$ \\
\hline $\begin{array}{l}\text { Saxagliptina } \\
\text { Saxagliptina } 2,5 \text { horas } \\
\text { Metabólito } 3,1 \text { horas }\end{array}$ & $\begin{array}{l}\text { Renal: } 60 \% \\
\text { (24\% na forma inalterada e } \\
36 \% \text { como metabólito) } \\
\text { Fecal: } 22 \%\end{array}$ & $\begin{array}{l}\text { Clearance < 50mL/min, em pa- } \\
\text { cientes em diálise "Usar } 2,5 \mathrm{mg} / \\
\text { dia" } \\
\text { Clearance > } 50 \mathrm{~mL} / \mathrm{min} \text { "Não é } \\
\text { necessário ajuste de dose" } \\
\text { Idosos sem doença renal "Não é } \\
\text { necessário ajuste de dose" }\end{array}$ & $\begin{array}{l}\text { Oral, } 2,5 \mathrm{mg} \text { a } 5 \mathrm{mg} \text { uma } \\
\text { vez ao dia }\end{array}$ \\
\hline $\begin{array}{l}\text { Linagliptina } \\
\text { Superior a } 100 \text { horas }\end{array}$ & $\begin{array}{l}\text { Renal: } 5 \%-7 \% \\
\text { Biliar: } 80 \%\end{array}$ & $\begin{array}{l}\text { Pode ser usada na doença re- } \\
\text { nal "Não necessita de ajuste de } \\
\text { dose". }\end{array}$ & $\begin{array}{l}\text { Oral, } 5 \mathrm{mg} \text { uma vez ao } \\
\text { dia }\end{array}$ \\
\hline $\begin{array}{l}\text { Alogliptina } \\
12 \text { a } 21 \text { horas }\end{array}$ & $\begin{array}{l}\text { Renal: } 76 \% \\
\text { (60\%-71\% na forma inalterada) } \\
\text { Fezes: } 13 \%\end{array}$ & $\begin{array}{l}\text { Clearance entre }<30 \mathrm{~mL} / \mathrm{min} \\
\text { "Usar } 6,25 \mathrm{mg} / \mathrm{dia} \text {, via oral" } \\
\text { Clearance entre } 30 \mathrm{~mL} / \mathrm{min}-60 \\
\mathrm{~mL} / \mathrm{min} \text { "Usar } 12,5 \mathrm{mg} / \mathrm{dia} \text {, via } \\
\text { oral" } \\
\text { Clearance > } 60 \mathrm{~mL} / \mathrm{min} \text { "Não ne- } \\
\text { cessita de ajuste de dose" }\end{array}$ & $\begin{array}{l}\text { Oral } 25 \mathrm{mg} \text { uma vez ao } \\
\text { dia }\end{array}$ \\
\hline
\end{tabular}


cont...

\begin{tabular}{|c|c|c|c|}
\hline \multicolumn{4}{|c|}{ Inibidores do cotransportador de sódio e glicose (iSGLT2) } \\
\hline $\begin{array}{l}\text { Dapagliflozina } \\
12,9 \text { horas }\end{array}$ & $\begin{array}{l}\text { Renal: } 75 \% \text { (menos que } 2 \% \text { na } \\
\text { forma inalterada) } \\
\text { Fecal: } 21 \% \text { ( } 15 \% \text { na forma inal- } \\
\text { terada) }\end{array}$ & $\begin{array}{l}\text { TFG }<60 \mathrm{~mL} / \mathrm{min} / 1,73 \mathrm{~m}^{2} \text { "Não } \\
\text { usar" } \\
\text { TFG }>60 \mathrm{~mL} / \mathrm{min} / 1,73 \mathrm{~m}^{2} \text { "Usar } \\
\text { até } 10 \mathrm{mg} " \\
\text { Hemodiálise "Não usar" } \\
\text { Idosos sem doença renal "Não é } \\
\text { necessário ajuste de dose" }\end{array}$ & $\begin{array}{l}\text { Oral, } 5 \text { mg uma vez ao } \\
\text { dia, pela manhã. } \\
\text { "A dose pode ser aumen- } \\
\text { tada para } 10 \mathrm{mg} \text { se ne- } \\
\text { cessário". }\end{array}$ \\
\hline $\begin{array}{l}\text { Canagliflozina } \\
10,6 \text { a } 13,1 \text { horas }\end{array}$ & $\begin{array}{l}\text { Renal: } 33 \% \\
\text { ( } 30,5 \% \text { como metabólitos e } \\
\text { menos que } 1 \% \text { na forma inal- } \\
\text { terada) Fecal: } 41,5 \% \text { (como } \\
\text { metabólitos) }\end{array}$ & $\begin{array}{l}\text { TFG < } 45 \mathrm{~mL} / \mathrm{min}-1,73 \mathrm{~m}^{2} \text { "Não } \\
\text { usar" } \\
\text { TFG entre } 45-60 \mathrm{~mL} / \mathrm{min} / 1,73 \mathrm{~m}^{2} \\
\text { "Usar dose de } 100 \mathrm{mg} / \text { dia" } \\
\text { TFG >60 mL/min/1,73 m² "Não é } \\
\text { necessário ajuste de dose" } \\
\text { Hemodiálise "Não usar" }\end{array}$ & $\begin{array}{l}\text { Oral, } 100 \text { mg uma vez ao } \\
\text { dia, antes da primeira re- } \\
\text { feição do dia. } \\
\text { "A dose pode ser aumen- } \\
\text { tada para } 300 \mathrm{mg} \text { se ne- } \\
\text { cessário". }\end{array}$ \\
\hline $\begin{array}{l}\text { Empagliflozina } \\
12,4 \text { horas }\end{array}$ & $\begin{array}{l}\text { Renal: } 54,4 \% \text { ( } 27,2 \% \text { na forma } \\
\text { inalterada) } \\
\text { Fecal: } 41,2 \% \\
\text { (maior parte na forma inaltera- } \\
\text { da) }\end{array}$ & $\begin{array}{l}\text { TFG < } 45 \mathrm{~mL} / \mathrm{min} / 1,73 \mathrm{~m}^{2} \text { "Não } \\
\text { usar" } \\
\text { TFG > } 45 \mathrm{~mL} / \mathrm{min} / 1,73 \mathrm{~m}^{2} \text { "Usar } \\
\text { de } 10 \mathrm{mg} \text { a } 25 \mathrm{mg} " \\
\text { Diálise "Não usar" } \\
\text { Idosos sem doença renal "Não é } \\
\text { necessário ajuste de dose" }\end{array}$ & $\begin{array}{l}\text { Oral, } 10 \text { mg uma vez ao } \\
\text { dia, pela manhã. } \\
\text { "A dose pode ser aumen- } \\
\text { tada para } 25 \mathrm{mg} \text { se ne- } \\
\text { cessário". }\end{array}$ \\
\hline \multicolumn{4}{|l|}{ Insulinas } \\
\hline $\begin{array}{l}\text { Insulinas (Geral) } \\
3 \text { a } 24 \text { horas }\end{array}$ & Renal: $30 \%-80 \%$ & $\begin{array}{l}\text { TFG }<10 \mathrm{~mL} / \mathrm{min} / 1,73 \mathrm{~m}^{2} \text { "Usar } \\
50 \% \text { da dose" } \\
\text { TFG entre } 10 \mathrm{~mL} / \mathrm{min} / 1,73 \mathrm{~m}^{2}-50 \\
\mathrm{~mL} / \mathrm{min} / 1,73 \mathrm{~m}^{2} \text { "Usar } 75 \% \text { da } \\
\text { dose" } \\
\text { TFG > } 50 \mathrm{~mL} / \mathrm{min} / 1,73 \mathrm{~m}^{2} \text { "Usar } \\
100 \% \text { da dose" }\end{array}$ & \\
\hline
\end{tabular}

Fonte: Cavanaugh (2007), Gilbert (2014), Hung et al. (2015), Neves et al. (2009, Micromedex® (2015) e Berns e Glickman (2015).

Nota: 1/2: meia-vida de eliminação em horas (h); TFG: Taxa de filtração glomerular; HbA1c: hemoglobina glicada; DRC: Doença Renal Crônica.

\section{Discussão}

O bom controle da glicemia ajuda a prevenir o aparecimento da albuminúria, podendo até mesmo reduzir a progressão da nefropatia diabética (EBOH; CHOWDHURY, 2015). A escolha do medicamento é passo fundamental e deve ser criteriosa, principalmente, quando se trata de populações especiais em que determinados medicamentos são contraindicados ou devem ser utilizados com cautela.

Diante dos resultados encontrados, observou-se que a maioria dos antidiabéticos determina uso cauteloso em idosos com DRC, pois a função renal diminuída provoca o acúmulo do medicamento na 
forma inalterada e/ou seus metabólitos e aumenta o risco de crises hipoglicêmicas, que, por sua vez, podem gerar danos cerebrais irreversíveis e alterações na circulação sanguínea (NERY, 2008). Os fatores que podem justificar o aumento desses riscos serão discutidos a seguir.

\section{1) Uso não recomendado}

Sulfonilureias

Clorpropramida possui metabolização hepática, gerando dois metabólitos ativos, podendo chegar a até $90 \%$ de excreção renal, além de ter meia-vida de eliminação longa. Diante disso, está associada a um maior risco de hipoglicemia, que pode ser acentuada em idosos com DRC, devido à diminuição da excreção renal, e consequentemente ao acúmulo de seus metabólitos ativos, que podem reduzir ainda mais os níveis de glicose no sangue, podendo ocasionar hipoglicemia grave (CAVANAUGH, 2007; GAME, 2014).

Diante dessas evidências, é importante destacar que a clorpropramida está inserida no critério de Beers, uma lista que contém medicamentos que são potencialmente inapropriados para idosos (AMERICAN GERIATRICS SOCIETY, 2015). Vale destacar que existem medicamentos da classe das sulfonilureias que podem ser utilizados com cautela e até mesmo alguns que podem ser utilizados com segurança.

\section{2) Uso restrito, a depender dos valores do clearance e/ou da taxa de filtração glomerular estimada do paciente}

Sulfonilureias

Glibenclamida e glimepirida são metabolizadas pelo fígado em metabólitos ativos e são eliminadas pelos rins, dessa forma, há uma maior chance de acúmulo desses metabólitos no organismo na vigência da DRC. A monitorização glicêmica torna-se necessária devido ao risco de hipoglicemia em idosos (GAME, 2014; NEVES et al., 2009). Pode haver necessidade de redução da dose desses medicamentos de acordo com o valor do clearance de cada paciente e quando o valor de creatinina for $>2 \mathrm{mg} / \mathrm{dL}$ (MICROMEDEX $^{\circledR}, 2015$ ).

\section{Glinidas}

Nateglinida possui meia-vida de eliminação mais longa que repaglinida e a maior parte de seus metabólitos é excretada pela urina. Portanto, deve ser utilizada com cautela e com monitorização de possíveis crises hipoglicêmicas para avaliação de necessidade de ajustes de dose em idosos com DRC (COELHO; CARRILHO; INCHAUSTEGUI, 2013).

Inibidores do cotransportador de sódio-glicose 2

A glicose é filtrada livremente pelos glomérulos e totalmente reabsorvida pelo túbulo proximal por meio do cotransportador de sódio-glicose. Ao bloquear esse transportador, essa classe de medicamentos aumenta a glicosúria 
e, com isso, reduz a glicemia (GILBERT, 2014). Na DRC, a redução da TFG diminui a quantidade de glicose eliminada na urina, reduzindo assim a efetividade do medicamento. $\mathrm{O}$ cálculo da TFG, antes da prescrição desses medicamentos, permite evitar prescrições irracionais (GAME, 2014).

\section{Biguanida}

A classe das biguanidas é representada pela metformina, considerada como tratamento de primeira escolha no caso de DM tipo 2 (SOCIEDADE BRASILEIRA DE DIABETES, 2015). A metformina é excretada pelos rins na forma inalterada, por isso a função renal é importante para determinar a segurança do medicamento (COELHO; CARRILHO; INCHAUSTEGUI, 2013).

Pode ser utilizada com segurança nas categorias 1 e 2 da DRC. Para pacientes que se encontram na categoria 3 , deve-se reduzir a dose pela metade, já nas categorias 4 e 5, não se deve utilizar esse medicamento. Outros autores preferem usar a creatinina como medida da função renal e contraindicam o uso da metformina caso a creatinina esteja maior que $1,4 \mathrm{mg} / \mathrm{dL}$ e $1,5 \mathrm{mg} / \mathrm{dL}$ em mulheres e homens, respectivamente. Deve-se evitar essa classe para idosos de 80 anos ou mais, exceto quando descartada a disfunção renal. Esses cuidados visam evitar a acidose lática, uma complicação rara, mas de grande mortalidade (CAVANAUGH, 2007; HUNG et al., 2015; NEVES et al., 2009).
Inibidores da dipeptidil peptidase 4 - iDPP4

Sitagliptina, vildagliptina, saxagliptina e alogliptina são excretadas pela urina na forma inalterada, como metabólitos inativos e ativos, dessa forma, o clearance do paciente deve ser analisado para ajuste de dose (COELHO; CARRILHO; INCHAUSTEGUI, 2013; NEVES et al., 2009).

Análogos de GLP-1 (glucagon-likepeptide 1)

Pacientes com DRC têm um aumento de meia-vida de eliminação de acordo com os estágios da doença em que se encontram. Exenatida pode ser usado em pacientes que se encontram nas categorias 1 a 3 , no entanto, naqueles em diálise, não foi bem tolerado, não sendo, então, indicado o uso (NEVES et al., 2009).

Pacientes que se encontram nas categorias 1 e 2 da doença podem usar liraglutida sem ajuste de dose, porém, não existem muitos estudos sobre seu uso em pacientes nas categorias 3 a 5 da doença, portanto, não se aconselha o uso (COELHO; CARRILHO; INCHAUSTEGUI, 2013; GAME, 2014). Para a lixisenatide, quando a Clearance estiver entre $30 \mathrm{~mL} /$ min e $50 \mathrm{~mL} / \mathrm{min}$, é necessário cautela ao utilizá-la (MICROMEDEX $\left.{ }^{\circledR}, 2015\right)$.

Inibidores da a-glicosidase

Acarbose é metabolizada pelo intestino dando origem a apenas um metabólito ativo, sendo menos de $2 \%$ excretado na urina. Dessa forma, pode ser usado em idosos com DRC, embora existam relatos de que nos pacientes em estágio 
avançado (categoria 4 e 5) da DRC ocorre acúmulo desse metabólito no organismo. Assim, o paciente deve ser monitorado, pois pouco se sabe sobre os efeitos tóxicos que o aumento dos níveis do fármaco e de seu metabólito pode causar. Pacientes com valores de creatinina $>2 \mathrm{mg} / \mathrm{dL}$ não devem utilizar esse medicamento (CAVANAUGH, 2007; NEVES et al., 2009).

Glitazona

A classe das glitazonas é representada pela pioglitazona, que é metabolizada pelo fígado e seu perfil farmacocinético independe da função renal. Portanto, não há necessidade de ajuste de dose em idosos com DRC, nem mesmo naqueles em terapia renal substitutiva (CAVANAUGH, 2007; COELHO; CARRILHO; INCHAUSTEGUI, 2013). Além disso, não há risco de hipoglicemia por não induzir a secreção de insulina (NEVES et al., 2009). No entanto, devido à retenção hídrica, já própria de estágios avançados da DRC, esse medicamento pode agravar quadro de insuficiência cardíaca congestiva (MICROMEDEX ${ }^{\circledast}, 2015$ ).

Insulina

O Instituto para Práticas Seguras no Uso dos Medicamentos considera a insulina um medicamento de risco, pois é um fármaco de estreita margem terapêutica e, dependendo da dose, pode causar hipoglicemia. Diante disso, é necessário o ajuste de dose em pacientes idosos com DRC, pois a insulina é excretada pelo rim e, nesses pacientes, ocorre menor eliminação da insulina e, consequentemente, aumento da meia vida de eliminação. Pacientes que se encontram nas categorias 3 a 5 da doença têm maior chance de apresentar hipoglicemia. Torna-se então necessária a monitorização glicêmica para o ajuste de dose de forma a prevenir a hipoglicemia, sobretudo no início do tratamento e/ou em caso de inserção de nova posologia (CAVANAUGH, 2007; INSTITUTO PARA PRÁTICA DE SEGURAS NO USO DE MEDICAMENTOS, 2013; SOCIEDADE BRASILEIRA DE DIABETES, 2015).

3) Medicamentos que podem ser utilizados por idosos com doença renal (sem necessidade de ajuste de dose)

Sulfonilureia

Dentre os medicamentos da classe das sulfonilureias, a gliclazida e a glipizida, são os medicamentos de escolha para idosos com DRC, pois apresentammeia vida de eliminação mais curta e os metabólitos gerados são inativos, fatores que minimizam o risco de hipoglicemia (CAVANAUGH, 2007; COELHO; CARRILHO; INCHAUSTEGUI, 2013).

Glinidas

Repaglinida não necessita de ajuste de dose, pois apenas $10 \%$ de seus metabólitos estão presentes na urina, além disso, são inativos. Deve-se preferir doses menores no início do tratamento, com aumento progressivo (NEVES et al., 2009). 
Inibidores da dipeptidil peptidase 4 - iDPP4

Linagliptina é excretada quase totalmente inalterada pela bile, portanto, pode ser usada nas categorias de 1 a 4 da DRC, porém, na categoria 5, quando há necessidade de terapia renal substitutiva, o medicamento deve ser usado com cautela (GAME, 2014).

Como todo artigo de revisão, este tem a limitação de utilizar dados secundários, assim, sua qualidade depende, também, dos artigos originais. Ademais, diversos medicamentos são novos e apresentam um número limitado de estudos, sobretudo com população idosa.

\section{Conclusão}

A presente revisão evidencia a importância da individualização do tratamento para idosos com DM e DRC e isso justifica a necessidade de atuação da equipe interdisciplinar no cuidado ofertado a esses pacientes, visto que, para esta individualização, são necessários conhecimentos específicos multidisciplinares.

Diante das evidências analisadas, observou-se que a escolha do antidiabético em idosos com DRC deve ser baseada no perfil farmacocinético de cada medicamento e em parâmetros clínicos e laboratoriais do paciente, como valores de creatinina, clearance e TFG estimada, visto que esses pacientes apresentam grau variável de eliminação dos fármacos e seus metabólitos.

O controle da glicemia é de grande importância para jovens e idosos, pois níveis glicêmicos constantemente eleva- dos causam danos ao organismo e esse descontrole resulta em maiores riscos de complicações micro e macrovasculares, danos em tecidos, perda da função normal, falência de vários órgãos e crises hipoglicêmicas, essa última mais acentuada em idosos.

A maior preocupação de uso dos antidiabéticos em idosos com DRC é o risco de acúmulo de fármaco e/ou metabólitos ativos no organismo, que, por sua vez, podem causar hipoglicemia, comprometendo a segurança dos pacientes. Crises hipoglicêmicas são causas frequentes de quedas, fraturas ósseas, hospitalizações e consultas em unidades de emergências.

\section{Hypoglicemic agents safety profile for use in elderly patients with Kidney disease}

\section{Abstract}

The prevalence of Diabetes mellitus (DM) increases with increasing age. In Brazil, $21.6 \%$ of the patients aged 65 or more have DM. DM is one of the most common causes of chronic kidney disease (CKD), when associated with ageing can worsen the clinical situation. Currently, there are several classes of antidiabetic, however the choice of medication for patients with CKD must be careful, especially in elderly patients. The objectives of this study are to analyze the safety profile of antidiabetic drugs in elderly patients with CKD, and categorize according to precaution of use. An individual analysis of pharmacokinetic profile of antidiabetics drugs and possible contraindications and restrictions of use in case of CKD was conducted. To search the information, we used Micromedex ${ }^{\circledR}$, and arti- 
cles in PubMed and SciELO databases. In regard of the findings, it was observed that the clorpropramida should be avoided by elderly patients with CKD. Others must be used with caution in accordance with the glomerular filtration rate (GFR) and creatinine clearance as nateglinide, liraglutide, exenatide, sitagliptin, vildagliptin, saxaglipitin, metformin, pioglitazone, acarbose, Insulin and Inhibitors of sodium-glucose co-transporter type 2 (SGLT2). Safe options considered are: gliclazide, glipizide, repaglinide and linagliptin. Therefore, the choice of antidiabetic drug for the elderly with CKD should be based on the pharmacokinetic profile of drugs and clinical and laboratory parameters of the patient.

Keywords: Diabetes mellitus. Diabetic nephropathies. Hypoglycemic agents. Kidney Failure Chronic.

\section{Agradecimentos}

À Universidade Federal de São João Del-Rei, pelo apoio estrutural.

\section{Referências}

AMERICAN GERIATRICS SOCIETY. Updated Beers criteria for potentially inappropriate medication use in older adults. Journal of the American Geriatrics Society, v. 63, n. 11, p. 1-20, nov. 2015.

BASTOS, M. G.; BREGMAN, R.; KIRSZTAJN, G. M. Doença renal crônica: frequente e grave, mas também prevenível e tratável. Revista da Associação Médica Brasileira, São Paulo (SP), v. 56, n. 2, p. 248-253, 2010.

BERNS, J. S.; GLICKMAN, J. D. Management of hyperglycemia in patients with type 2 diabetes and pre-dialysis chronic kidney disease or end-stage renal disease. UpToDate, 2015.
BORGES, G. M.; CAMPOS, M. B.; SILVA, L. G. C. Transição na estrutura etária do Brasil: oportunidades e desafios para a sociedade nas próximas décadas. In: ERVATTI, L. R.; BORGES, G. M.; JARDIM, A. P. Mudança demográfica do Brasil no início do século $X X I$ : subsídios para as projeções da população. Rio de Janeiro: IBGE, 2015. p. 138-151. (Estudos e Análises. Informação demográfica e socioeconômica, n. 3). Disponível em: <http://biblioteca.ibge.gov.br/visualizacao/ livros/liv93322.pdf>. Acesso em: out. 2015.

CAVANAUGH, K. L. Diabetes management issues for patients with Chronic Kidney Disease. Clinical Diabetes, v. 25, n. 3, p. 90-97, Jul. 2007.

COELHO, S.; CARRILHO, P.; INCHAUSTEGUI, L. Management of hyperglycaemia in type 2 diabetic patients with Chronic Kidney Disease. Portuguese Journal of Nephrology \& Hypertension, Odivelas, v. 27, n. 2, p. 91100, 2013.

EBOH, C.; CHOWDHURY, T. A. Management of diabetic renal disease. Annals of Translational Medicine, Hong Kong, v. 3, p. 2305-5839, 2015.

GAME, F. Novel hypoglycemic agents: considerations in patients with Chronic Kidney Disease. Nephron Clinical Practice, v. 126, n. 1, p. 14-18, 2014.

GILBERT, R. E. SGLT-2 inhibition in patients with kidney disease. Portuguese Journal of Endocrinology, Diabetes and Metabolism, v. 40, n. 6, p. 23-27, 2014.

GREENSPAN, F.; STREWLER G. Endocrinologia Básica \& Clínica. 7. ed. Rio de Janeiro: Guanabara Koogan, 2006. p. 599.

HUNG, S. C. et al. Metformin use and mortality in patients with advanced chronic kidney disease: national, retrospective, observational, cohort study. The Lancet Diabetes \& Endocrinology, v. 8587, n. 15, p. 1-10, 2015. 
INSTITUTO PARA PRÁTICAS SEGURAS NO USO DE MEDICAMENTOS. Medicamentos potencialmente perigosos. Boletim ISMP, Belo horizonte, v. 2, p. 1-3, mar. 2013.

KIDNEY DISEASE IMPROVING GLOBAL OUTCOMES. Clinical Practice Guideline for the Evaluation and Management of Chronic Kidney Disease. Kidney International Supplements. v. 3,n. 1, p. 1-150, 2013.

LEMOS, J. R. D. et al. O envelhecimento como fator determinante nas modificações da estrutura renal. Publicatio - Universidade Estadual de Ponta Grossa Ciências Biológicas e da Saúde, Ponta Grossa (PR), v. 14, n. 1. p. 41-51, mar. 2008.

MICROMEDEX SOLUTION. Evidence-Based Clinical Decision Support. Disponível em: <http://micromedex.com>. Acesso em: out. 2015.

NERY, M. Hipoglicemia como fator complicador no tratamento do diabetes melito tipo 1. Arquivos Brasileiros de Endocrinologia Metabólica, São Paulo (SP), v. 52, n. 2, mar. 2008.

NEVES, M. D. F. et al. Tratamento da hiperglicemia nos pacientes com diabete melito e doença renal crônica. Jornal Brasileiro de Nefrologia, São Paulo (SP), v. 31, n. 1, p. 21-27, 2009.

OBRELI NETO, P. R.; BALDONI, A. O.; GUIDONI, C. M. Farmacoterapia: guia terapêutico de doenças mais prevalentes. Diabetes mellitus. São Paulo: Pharmabooks, 2013. p. 58-112.

SHIH, C. J. et al. Association of hypoglycemia with incident chronic kidney disease in patients with type 2 Diabetes. Medicine, Baltimore, v. 94, n. 16, p. 771-779, 2015.

SOCIEDADE BRASILEIRA DE DIABETES. Diretrizes da Sociedade Brasileira de Diabetes: 2014-2015. São Paulo: AC Farmacêutica, 2015. Disponível em: <http://www.diabetes. org.br/novas-diretrizes-da-sociedade-brasileira-de-diabetes>. Acesso em: out. 2015.
SOCIEDADE BRASILEIRA DE ENDOCRINOLOGIA E METABOLOGIA. Vigitel 2011: diabetes. 2011. Disponível em: <http://www. endocrino.org.br/vigitel-2011-diabetes/>. Acesso em: set. 2015.

SOCIEDADE BRASILEIRA DE NEFROLOGIA. Diretrizes da Amb: insuficiência renal aguda. 2007. Disponível em: <http:// sbn.org.br/app/uploads/Diretrizes_Insuficiencia_Renal_Aguda.pdf>. Acesso em: nov. 2016.

Insuficiência renal. 2011. Disponível em: <http://www.sbn.org.br/publico/ insuficiencia-renal>. Acesso em: out. 2015.

TONELLI, M.; RIELLA, M. C. Doença renal crônica e o envelhecimento da população. Jornal Brasileiro de Nefrologia, São Paulo (SP), v. 36, n. 1, p. 1-5, 2014. 